\title{
Stress amplifies sex differences in primate prefrontal profiles of gene expression
}

\author{
Alex G. Lee ${ }^{1}$, Megan Hagenauer ${ }^{2}$, Devin Absher ${ }^{3}$, Kathleen E. Morrison ${ }^{4}$, Tracy L. Bale ${ }^{4}$, Richard M. Myers ${ }^{3}$, \\ Stanley J. Watson ${ }^{2}$, Huda Akil ${ }^{2}$, Alan F. Schatzberg ${ }^{1}$ and David M. Lyons ${ }^{1 *}$
}

\begin{abstract}
Background: Stress is a recognized risk factor for mood and anxiety disorders that occur more often in women than men. Prefrontal brain regions mediate stress coping, cognitive control, and emotion. Here, we investigate sex differences and stress effects on prefrontal cortical profiles of gene expression in squirrel monkey adults.

Methods: Dorsolateral, ventrolateral, and ventromedial prefrontal cortical regions from 18 females and 12 males were collected after stress or no-stress treatment conditions. Gene expression profiles were acquired using HumanHT-12v4.0 Expression BeadChip arrays adapted for squirrel monkeys.

Results: Extensive variation between prefrontal cortical regions was discerned in the expression of numerous autosomal and sex chromosome genes. Robust sex differences were also identified across prefrontal cortical regions in the expression of mostly autosomal genes. Genes with increased expression in females compared to males were overrepresented in mitogen-activated protein kinase and neurotrophin signaling pathways. Many fewer genes with increased expression in males compared to females were discerned, and no molecular pathways were identified. Effect sizes for sex differences were greater in stress compared to no-stress conditions for ventromedial and ventrolateral prefrontal cortical regions but not dorsolateral prefrontal cortex.
\end{abstract}

Conclusions: Stress amplifies sex differences in gene expression profiles for prefrontal cortical regions involved in stress coping and emotion regulation. Results suggest molecular targets for new treatments of stress disorders in human mental health.

Keywords: Social stress, Sex biased, Prefrontal cortex, Gene expression profiling, Neurotrophins, MAPK signaling, Squirrel monkeys

\section{Background}

Mood and anxiety disorders occur more often in women than men [1-4]. These mental health disorders are associated with stress-induced modifications in prefrontal cortex [5-7]. Neuroscience research has until recently focused on males [8], and sex differences in stress effects on primate prefrontal profiles of gene expression are not known. Differential gene expression profiles have been reported for diverse brain regions in humans $[9,10]$ and various monkey species $[11,12]$, but comparisons across prefrontal cortical regions in primates are uncommon. Prefrontal regions are of interest as elements in brain circuits

\footnotetext{
* Correspondence: dmlyons@stanford.edu

'Department of Psychiatry and Behavioral Sciences, Stanford University, 1201

Welch Rd MSLS Room P104, Stanford, CA 94305-5485, USA

Full list of author information is available at the end of the article
}

that mediate stress coping, cognitive control, and emotion [13-16]. Human prefrontal neurobiology is modeled more often in nonhuman primates compared to other animals because of species differences in this region of brain [5].

Sex differences in gene expression profiles were initially thought to be limited in human brain [17]. Recent evidence suggests that many genes may be differentially expressed between the sexes for various brain regions in mice [18-20], monkeys [21], and humans [9, 22, 23]. Certain sex differences may reflect species-specific adaptations [24], but sex differences in stress neurobiology commonly occur in many mammals [25] including squirrel monkeys [26]. Sex differences and stress effects in squirrel monkey prefrontal cortical profiles of gene expression may provide insights on the biology of sex differences in human mental health. 
Here, we examine prefrontal cortical gene expression profiles in female and male squirrel monkey adults randomly selected from a larger sample randomized to social separation stress or no-stress treatment conditions. Social separations consistently increase plasma levels of the stress hormone cortisol in female [27] and male [28] squirrel monkeys. Both sexes respond to separations from same-sex conspecifics with prolonged increases in cortisol, but females and males have not been directly compared. In separate studies, females show diminished neuroendocrine stress responses [29] and males show increased hippocampal neurogenesis [30] measured 24 weeks after exposure to same-sex social separations. Long-term effects are likewise assessed here for prefrontal cortical profiles of gene expression in both sexes. Gene expression profiling represents discovery research [31] that is not guided by hypotheses about specific genes. Analyses of gene expression profiles demand statistical sophistication [32], but we aim to balance statistics with biological implications in this report.

\section{Methods}

Twenty two male and 48 female squirrel monkey (Saimiri sciureus) adults served as subjects. These monkeys were approximately 6-17 years of age determined from birth records or estimated from dentition. All monkeys were housed in climate controlled rooms at $\sim 26^{\circ} \mathrm{C}$ on 12:12-h light/dark cycles with lights on at 07:00. Cages were cleaned daily and provisioned with fresh drinking water, commercial monkey chow, and a variety of fruit and vegetable supplements. Various toys, swinging perches, and simulated foraging activities were provided for environmental enrichment.

In naturalistic and semi-naturalistic conditions, squirrel monkeys live in sexually segregated groups comprised of multiple females and many fewer males [33]. To simulate this social organization in our control condition, we randomly assigned 10 males to live with a familiar same-sex conspecific in undisturbed pairs and 24 females to live in undisturbed groups each comprised of four familiar same-sex conspecifics. Another agematched sample of 12 males and 24 females were randomized to the stress treatment condition in which monkeys housed as described above were intermittently removed from same-sex conspecifics at 12-15-week intervals for 6-7 repeated social separations. During each 3-week separation session, monkeys were housed individually in cages that allowed visual, auditory, olfactory, and limited tactile contact between adjacent animals.

\section{Prefrontal brain tissue collection}

Brains from randomly selected monkeys in the stress ( $n$ $=6$ males and 6 females) and no-stress $(n=6$ males and 12 females) conditions were collected 4 weeks after completion of the treatment conditions when all monkeys were socially housed with same-sex conspecifics. Collection time was selected to assess long-term effects of stress. After euthanasia, brains were collected using established procedures [34] between 08:00 and 10:00 to control for circadian effects. All brains were collected during nonbreeding seasons when circulating sex steroid hormones remain stable at low levels in these seasonally breeding primates [35].

Hemisected brains were cut into blocks, flash frozen in isopentane at $-20{ }^{\circ} \mathrm{C}$, and stored at $-80{ }^{\circ} \mathrm{C}$. Fresh frozen blocks from the left brain side immediately anterior to the corpus callosum were cut on a cryostat into $20 \mu \mathrm{m}$ coronal sections at $-18{ }^{\circ} \mathrm{C}$ using RNase-free methods. Serial sections were mounted on glass slides and then stored at $-80{ }^{\circ} \mathrm{C}$. Dorsolateral, ventrolateral, and ventromedial prefrontal cortical regions depicted in Fig. 1 were dissected from coronal sections at $0{ }^{\circ} \mathrm{C}$ under a stereo-zoom microscope using RNAse-free instruments and a squirrel monkey brain atlas [36]. All three prefrontal cortical regions were dissected from the same three tissue sections randomly selected from $\sim 100$ serial tissue sections acquired from each monkey anterior to the corpus callosum on the left brain side. Tissues collected from separate sections were combined into a single sample for each region per monkey.

\section{BeadChip array hybridization}

Tissue samples were homogenized with a motorized pellet pestle, and total RNA was extracted using AllPrep DNA/RNA Micro kits (Qiagen). Samples were amplified (RiboAmp Plus 1.5-round RNA Amplification, Applied Biosystems) for the production of biotinylated cRNA (Bioarray High Yield RNA Labeling, ENZO) and

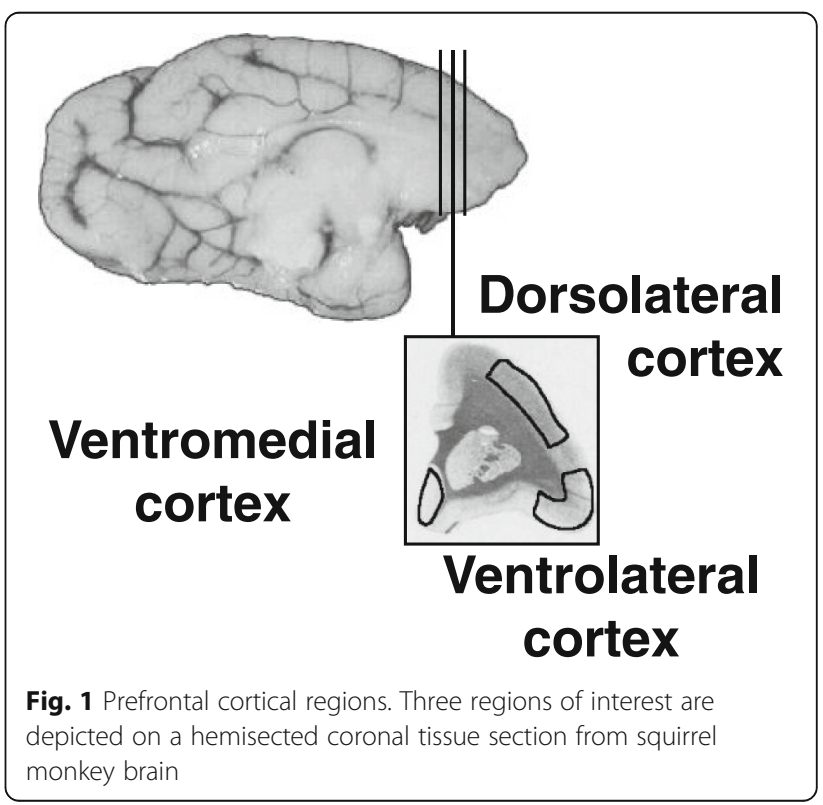


subsequently hybridized to HumanHT-12v4.0 Expression BeadChip arrays (Illumina) that were scanned on a BeadStation system following manufacturer's instructions. A total of 90 BeadChip arrays were used to generate gene expression profiles for dorsolateral, ventrolateral, and ventromedial prefrontal cortex from 18 females and 12 males. No pooling of samples across monkeys or regions was necessary. Prefrontal cortical regions, sex, and stress treatment conditions were randomly counterbalanced across separate BeadChip arrays.

\section{qPCR validation}

To verify array results, we focused on HSD11B1 and CACNG2 expression in each of the three prefrontal cortical regions assessed by quantitative real-time polymerase chain reaction (qPCR) with $A C T B$ as the reference gene. Primers designed with Primer-BLAST (NCBI) for Sybr Green assays with parameters set as described elsewhere [37] are as follows: HSD11B1 forward 5' AT GTGGTGGTGACAGCGAG 3'; HSD11B1 reverse 5' T ATAGTGCGCTGAGGCTGCT 3'; CACNG2 forward 5' GGCCCTGTCCTTCATCATC 3'; CACNG2 reverse 5' GCAGAGGCCTGGAGGTAGT 3'; ACTB forward 5' C AAGGCCAATCGTGAGAAGA $3^{\prime}$; and $A C T B$ reverse 5' AGAGGCGTACAAGGAAAGCA 3'. The same samples analyzed by array were used for qPCR reactions conducted following manufacturer's instructions (Biorad, SsoAdvanced Universal SYBR Green) under these conditions: $250 \mathrm{nM}$ primers, $50 \mathrm{mM} \mathrm{Na}^{+}, 3 \mathrm{mM} \mathrm{Mg}^{++}$, and $1.2 \mathrm{mM}$ dNTP, using the MxPro3000 (Stratagene). Results from qPCR were analyzed as described elsewhere [38].

\section{Array data processing}

Each BeadChip array has more than 47,000 probes designed for the human genome. To identify probes suited for squirrel monkeys, we compared probe sequences against the squirrel monkey genome (Broad Institute, GCA_000235385.1) using BLAT [39]. Selected probes were required to match a single continuous segment of the squirrel monkey genome at $95 \%$ homology or greater. For the 11,209 selected probes, expression values were imported into $R$ [40], background corrected using maximum likelihood estimation [41], log2 transformed, and quantile normalized. Normalized data were analyzed for outliers using box plots, sample-sample correlation matrices, and principle components analysis. Two males from the stress condition were excluded as outliers with a median sample-sample correlation of 0.946 compared to 0.980 for all remaining samples. Probes not associated with known genes were discarded. Using these procedures, we analyzed 8853 probes interrogating 7299 unique genes in 18 females and 10 males for 3 prefrontal cortical regions.

\section{Statistical analysis}

A multi-level regression model also known as a hierarchical linear model or a mixed-effects model [42] was used to assess stress, sex, and prefrontal cortical region main effects in expression data for each probe. These analyses were performed in $\mathrm{R}$ ( $\mathrm{R}$ v.3.3.0: https://cran.rproject.org, RStudio v.0.99.896: http://www.rstudio.com/ ) using the $\operatorname{lme}()$ function in the nlme package (https:// cran.r-project.org/package $=$ nlme) with a default correlation structure (no within-group correlation) and method (REML: maximizing the restricted log-likelihood). Our model included the fixed effects of stress (reference level $=$ control condition), sex (reference level $=$ female), and prefrontal region (reference level $=$ dorsolateral prefrontal cortex) with repeated measures accounted for by including individual subjects as a random effects variable (random intercept). Age was unevenly distributed across stress and sex groupings, and in humans, age alters frontal cortical gene expression profiles [43]. We therefore included age class as a fixed effect covariate in our model for squirrel monkeys (younger adult $=-1$, middle-age adult $=0$, older adult $=1$ ). Age class categories were used to accommodate age estimates based on dentition. All test statistics were evaluated with two-tailed probabilities, and Benjamini-Hochberg false discovery rate corrected $q$ values were computed for each nominal $P$ value to address the multiple comparisons issue [44].

To further explore regional differences in gene expression profiles, a single summary statistic for regional effects on expression of each probe was extracted using a likelihood ratio approach. Specifically, we used anova() function in $\mathrm{R}$ to compare the full multilevel model described above with a model that lacked the fixed effect term for region [45]. For probes with significant regional effects $(P<0.05)$ that survived multiple testing correction $(q<0.05)$, hierarchical cluster analysis with average linkage was used to assess relations between regions in SYSTAT13 (http://systatsoftware.com/).

We also examined how regional differences in gene expression profiles of prefrontal cortex in squirrel monkeys compare to humans. To identify regions of human prefrontal cortex with gene expression profiles similar to regions of squirrel monkey prefrontal cortex, we compared monkey results to Allen Brain Atlas human brain microarray data (http://human.brain-map.org, downloaded 12/2015). Allen Brain Atlas microarray data span 160 cortical and subcortical brain regions in high-quality post-mortem tissue from healthy adult men and women [46]. Preprocessed microarray data from the Allen Brain Atlas website were downloaded as $z$-scores with $\log 2$ expression data for each probe transformed so that the mean for the entire brain was equal to 0 and the standard deviation equal to 1 . For our purposes, we then extracted human expression data from 
20 specific regions of frontal or anterior cingulate cortex listed in Additional file 1: Table S1 and re-centered and rescaled these data for each probe. We also centered and scaled our squirrel monkey data for each probe to make similar formats for monkeys and humans, and averaged data for each probe by region. Average regional gene expression signatures for both species were then compared using a region-region correlation matrix and hierarchical clustering.

To identify molecular pathways in monkey gene expression data, probes with significant sex differences $(P$ $<0.05)$ that survived multiple testing correction $(q<$ 0.05) were uploaded to DAVID6.7 [47] for annotation with Kyoto Encyclopedia of Genes and Genomes (KEGG) terms. The complete set of 8853 probes that targeted 7299 unique genes identified as suitable for squirrel monkeys served as background for all pathway analyses. Chromosome locations are unknown for many squirrel monkey genes, and therefore we used a comparative approach with Ensembl BioMart to identify genes on sex chromosomes in humans and marmoset monkeys (Callithrix jacchus, C_jacchus3.2.1). Like squirrel monkeys, marmosets are also New World primates. Effect sizes for sex differences in gene expression were calculated using Eta squared $\eta^{2}[48]$ and assessed by analysis of variance in SYSTAT13 with stress as a between-subjects factor and region considered a withinsubjects factor.

\section{Results}

Age class effects did not survive multiple testing correction for any of the 8853 probes that targeted 7299 unique genes. None of the probes likewise survived multiple testing correction for interactions between stress, sex, and prefrontal cortical regions in expanded models. Therefore, we focus on main effects illuminated by two primary gradients in the data identified by multidimensional scaling (Fig. 2).

\section{Regional effects}

Prefrontal cortical region main effects were discerned for 5884 probes that targeted 5155 unique genes $(2.6 \times$ $10^{-33} \leq P \leq 0.05$ ). Nominal $P$ values for 5557 of these probes that targeted 4905 genes survived multiple testing correction $\left(2.3 \times 10^{-29} \leq q \leq 0.05\right.$; Additional file 2: Table S2). More than 240 probes with region main effects $(P<0.05)$ that survived multiple testing correction $(q<0.05)$ targeted X chromosome genes in humans and marmoset monkeys (Additional file 2: Table S2). None of the probes in our gene expression profiling analyses of squirrel monkeys targeted $\mathrm{Y}$ chromosome genes in humans or marmosets.

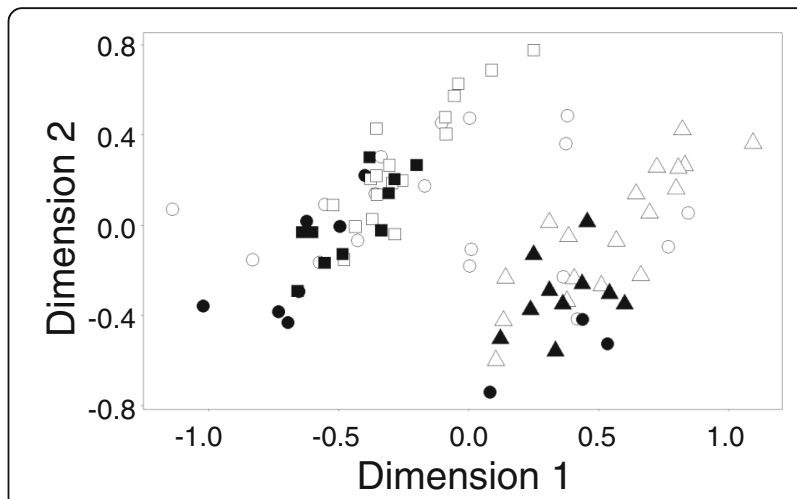

Fig. 2 Two primary gradients identified by multi-dimensional scaling of array data. Dimensions 1 and 2 reflect differences between the sexes and variation between prefrontal cortical regions. Females (white shapes) are primarily in the upper right with males (black shapes) in the lower left. Ventrolateral prefrontal cortex (triangles) is primarily in the lower right with dorsolateral (circles) and ventromedial (squares) prefrontal cortical regions in the upper left

Hierarchical cluster analysis of probes with prefrontal region main effects $(P<0.05)$ that survived multiple testing correction $(q<0.05)$ revealed that ventromedial and dorsolateral regions were more similar to one another compared to ventrolateral prefrontal cortex (Fig. 3). The number of probes differentiating prefrontal cortical regions in within-subjects pairwise comparisons supported the conclusion that ventromedial and dorsolateral regions resembled one another more than ventrolateral prefrontal cortex. Ventromedial and dorsolateral regions differed on 1302 probes $(P<0.05, q<0.05)$. Using the same pairwise approach, more than three times as many probes differed between ventrolateral prefrontal cortex compared to either ventromedial (4879 probes) or dorsolateral (4346 probes) prefrontal cortical regions $(P<0.05, q<0.05)$.

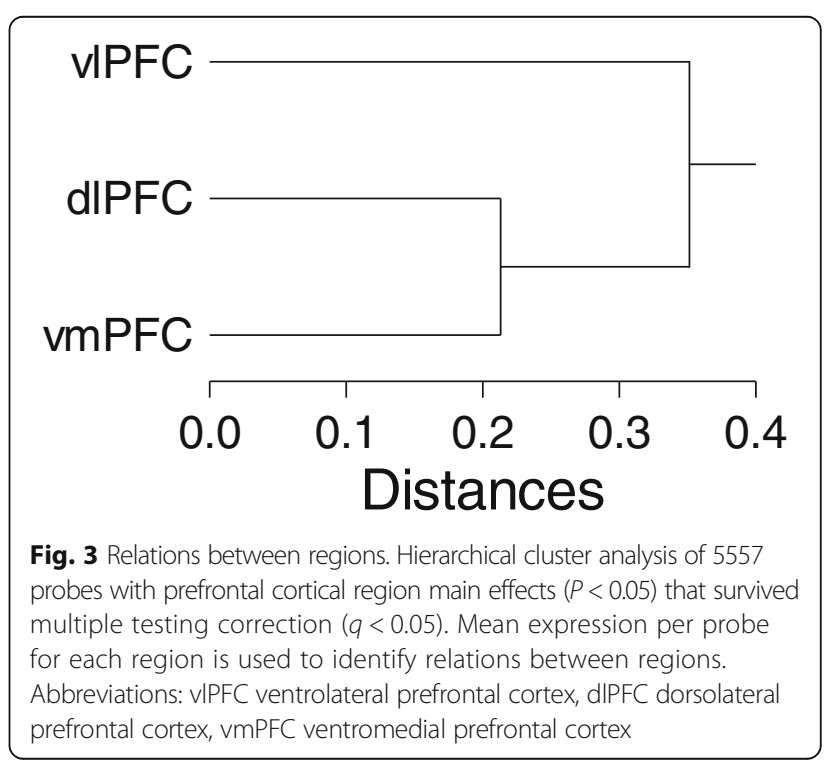


Cross-species comparisons indicated that squirrel monkey dorsolateral prefrontal cortex resembled middle frontal gyrus $\left(R=0.197, P=4.98 \times 10^{-87}\right)$ and precentral gyrus $\left(R=0.183, P=1.06 \times 10^{-74}\right)$ in humans portrayed in Allen Brain Atlas microarray data. Squirrel monkey ventromedial prefrontal cortex resembled parolfactory (or subcallosal) gyri $\left(R=0.111, P=1.71 \times 10^{-28}\right)$ and gyrus rectus $\left(R=0.107, P=2.00 \times 10^{-26}\right)$ in humans. Squirrel monkey ventrolateral prefrontal cortex resembled posterior orbital gyrus $\left(R=0.047, P=2.49 \times 10^{-6}\right)$ and frontal pole $\left(R=0.063, P=2.49 \times 10^{-6}\right)$ in humans.

\section{Sex effects}

Sex difference main effects in squirrel monkeys were discerned for 1270 probes that targeted 1219 unique genes $\left(8.5 \times 10^{-8}<P<0.05\right)$. Nominal $P$ values for 82 of these probes that targeted 80 unique genes survived multiple testing correction $\left(3.8 \times 10^{-4} \leq q \leq 0.05\right.$; Additional file 3: Table S3). Of these 82 probes, 19 had nominally significant age class effects $(P<0.05)$ that did not survive multiple testing correction (Additional file 3: Table S3). For these 19 probes, unbalanced age distributions could potentially contribute to differences between the sexes but this possibility does not likely explain sex differences for the remaining 63 probes.

Most genes expressed differently between the sexes across squirrel monkey prefrontal cortical regions did not reside on sex chromosomes. None of the probes targeted $\mathrm{Y}$ chromosome genes and only 2 of 82 probes with sex difference main effects $(P<0.05)$ that survived multiple testing correction $(q<0.05)$ targeted X chromosome genes in humans and marmoset monkeys. Both of these genes (SLC25A5 and TSC22D3) were expressed at higher levels in squirrel monkey females compared to males across all three prefrontal cortical regions.

Many probes expressed at higher levels in squirrel monkey females compared to males targeted autosomal genes. For example, HSD11B1 is located on chromosome 1 in humans and this gene was expressed at higher levels in squirrel monkey females compared to males across prefrontal cortical regions discerned by two probes on the array (Additional file 3: Table S3). Array results for both probes correlated with HSD11B1 expression determined by qPCR (Additional file 4: Table S4) and qPCR determinations were greater in females compared to males $(F(1,24)=17.2, P<0.001$ for the sex main effect). A region main effect was also discerned by qPCR $(F(2,48)=21.3, \quad P<0.001)$ and by both array probes (Additional file 2: Table S2) for HSD11B1 expression. Regional differences do not likely explain correlations because qPCR correlated with array measures for HSD11B1 regardless of whether prefrontal cortical regions were analyzed separately or combined (Additional file 4: Table S4). Comparable correlations were also identified for another autosomal gene (CACNG2 in Additional file 4: Table S4) that differed significantly between prefrontal cortical regions determined by array (Additional file 2: Table S2) and by qPCR $(F(2,48)=6.8, P=0.002$ for the region main effect).

Of the 82 probes with sex difference main effects $(P<$ $0.05)$ that survived multiple testing correction $(q<0.05)$, $64(78 \%)$ were increased across prefrontal cortical regions in squirrel monkey females compared to males. Conversely, 18 of 82 probes (22\%) were increased across prefrontal cortical regions in males compared to females. Molecular pathways of genes targeted by probes with sex difference main effects were assessed using DAVID6.7 [47].

For genes with increased expression in females compared to males, two KEGG pathway terms had nominal $P$ values that survived multiple testing correction (hsa04722 neurotrophin signaling pathway, 7.7-fold enrichment, $P=6.0 \times 10^{-4}, q=0.017$; and hsa04010 MAPK signaling pathway, 5.5-fold enrichment, $P=2.1 \times 10^{-4}, q$ $=0.012$ ). More than half of the genes identified in these pathways were classified under both neurotrophin and MAPK signaling (Table 1). No KEGG terms survived multiple testing correction for genes with increased expression in males compared to females.

To test for greater variation in gene expression associated with estrous cycle-related hormonal fluctuations, we followed a statistical approach described elsewhere by other investigators [49]. Coefficients of variation (CV) were calculated separately for no-stress control females and control males for each of the 82 probes with sex difference main effects $(P<0.05)$ that survived multiple testing correction $(q<0.05)$. Twelve females from the control condition were examined to increase chances of assessing different stages of the estrous cycle. Sexspecific CVs were not significantly greater in females compared to males for any prefrontal cortical region.

\section{Stress effects}

None of the stress main effects nor interactions survived multiple testing correction. Nevertheless, effect sizes for sex differences were greater in stress compared to nostress conditions (Fig. 4) as discerned by a stress main effect $\left(F(1,162)=46.3, P=1.9 \times 10^{-10}\right)$ and a stress-byregion interaction $\left(F(2,324)=26.5 P=2.1 \times 10^{-11}\right)$. Effect sizes for sex differences were greater in stress versus nostress conditions for ventromedial $(F(1,162)=63.1, P=$ $\left.2.4 \times 10^{-11}\right)$ and ventrolateral $(F(1,162)=43.2, P=6.5 \times$ $10^{-11}$ ) prefrontal cortical regions but not dorsolateral prefrontal cortex $(P=0.687)$. Three of 82 probes with sex difference main effects $(P<0.05)$ that survived multiple testing correction $(q<0.05)$ showed a nominally significant stress main effect $(P<0.05)$ but none of the stress effects survived multiple testing correction. 
Table 1 Genes in two molecular pathways. Specific genes overrepresented in neurotrophin and MAPK signaling pathways with greater expression in squirrel monkey females compared to males (mean \pm SEM) across prefrontal cortical regions

\begin{tabular}{|c|c|c|c|c|c|}
\hline KEGG pathway & Gene & Females & Males & $P$ & 9 \\
\hline Neurotrophin signaling & CAMK2G & $7.63 \pm 0.08$ & $7.16 \pm .012$ & 0.00001 & 0.009 \\
\hline \multirow[t]{3}{*}{ MAPK signaling } & TGFBR2 & $8.77 \pm 0.08$ & $8.24 \pm .011$ & 0.00044 & 0.048 \\
\hline & STK4 & $7.94 \pm 0.07$ & $7.38 \pm 0.10$ & 0.00006 & 0.016 \\
\hline & MAP3K4 & $8.31 \pm 0.08$ & $7.87 \pm 0.12$ & 0.00001 & 0.009 \\
\hline \multirow[t]{5}{*}{ Neurotrophin and MAPK signaling } & TRAF6 & $6.54 \pm 0.06$ & $6.19 \pm 0.09$ & 0.00006 & 0.016 \\
\hline & CRKL & $11.88 \pm 0.05$ & $11.58 \pm 0.06$ & 0.00010 & 0.020 \\
\hline & RPS6KA2 & $11.85 \pm 0.05$ & $11.55 \pm 0.06$ & 0.00009 & 0.020 \\
\hline & MAP2K5 & $5.93 \pm 0.06$ & $5.72 \pm 0.07$ & 0.00029 & 0.038 \\
\hline & MAPK10 & $12.09 \pm 0.04$ & $11.82 \pm 0.05$ & 0.00002 & 0.012 \\
\hline
\end{tabular}

The top 25 genes with the largest disparity in effect sizes for sex differences in stress versus no-stress conditions in ventromedial and ventrolateral prefrontal cortical regions are depicted in Table 2. Three of these genes (MAP3K4, TRAF6, CRKL) were identified earlier in MAPK and neurotrophin signaling pathways (Table 1). Another three genes (SLC6A1, SPRY4, TSC22D3) with large disparities in effect sizes for sex differences in stress versus no-stress conditions are discussed below.

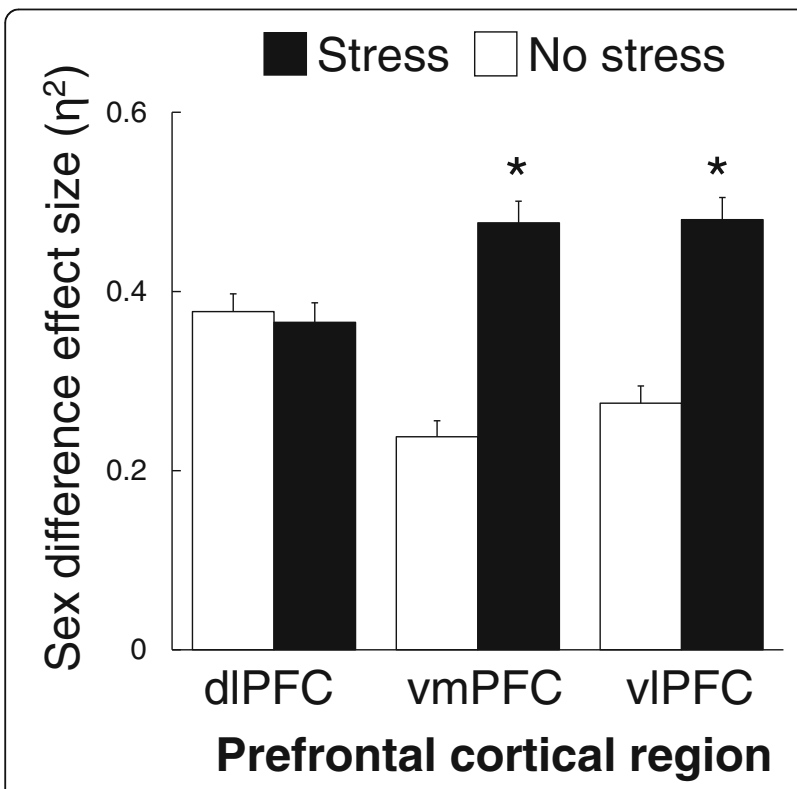

Fig. 4 Certain sex differences are amplified by stress. Effect sizes $\left(\eta^{2}\right)$ for sex differences are presented in stress and no-stress conditions for 82 probes that targeted 80 unique genes expressed in prefrontal cortical regions (mean $\pm \mathrm{SEM}$ ). The stress-by-region interaction described in the text reflects stress induced disparities in sex difference effect sizes for ventromedial and ventrolateral regions $\left({ }^{*} P<0.001\right)$ but not dorsolateral prefrontal cortex. Abbreviations: dIPFC dorsolateral prefrontal cortex, vmPFC ventromedial prefrontal cortex, vIPFC ventrolateral prefrontal cortex
Five of the 25 genes in Table 2 (MAP3K4, KCNIP1, HHIP, SLC24A2, TRIM4) had nominally significant age class effects $(P<0.05)$ that did not survive multiple testing correction (Additional file 3: Table S3). For these 5 genes, unbalanced age distributions could potentially contribute to stress related disparities in effect sizes for sex differences but this possibility is not likely for the remaining 20 genes $(80 \%)$ depicted in Table 2.

\section{Discussion}

We examined gene expression profiles for dorsolateral, ventrolateral, and ventromedial prefrontal cortical regions from female and male squirrel monkey adults randomly selected from a larger sample randomized to stress or no-stress conditions. Human-specific probes were aligned to the monkey genome and those with $\geq$ 95\% sequence homology were selected for analysis. Results indicate extensive variation between prefrontal cortical regions in the expression of numerous autosomal and sex chromosome genes. Robust sex differences were also discerned across prefrontal cortical regions in the expression of mostly autosomal genes. Genes with increased expression in females compared to males were overrepresented in MAPK and neurotrophin signaling pathways. Many fewer genes with increased expression in males compared to females were discerned, and no molecular pathways were identified. Effect sizes for sex differences were greater in stress compared to no-stress conditions for ventromedial and ventrolateral prefrontal cortical regions but not dorsolateral prefrontal cortex.

Differential gene expression profiles have been reported for diverse brain regions in humans $[9,10]$ and various monkey species $[11,12]$ but comparisons between primate prefrontal cortical regions are uncommon. We found that gene expression profiles for prefrontal cortical regions in squirrel monkeys resembled profiles of anatomically comparable prefrontal regions in humans. Squirrel monkey dorsolateral and 
Table 2 Top 25 genes with the largest disparity in effect sizes for sex differences in stress versus no-stress conditions. Effect sizes $\left(\eta^{2}\right)$ for sex differences in stress and no-stress conditions are presented for ventrolateral (vIPFC) and ventromedial (vmPFC) prefrontal cortical regions. $F>M$ signifies genes with greater expression in females compared to males, and $M>F$ signifies the reverse

\begin{tabular}{|c|c|c|c|c|c|c|}
\hline \multirow[t]{2}{*}{ Gene } & \multirow[t]{2}{*}{ Biological function } & \multicolumn{2}{|l|}{ VIPFC } & \multicolumn{2}{|c|}{ vmPFC } & \multirow[t]{2}{*}{ Effect direction } \\
\hline & & Stress & No-stress & Stress & No-stress & \\
\hline MAP3K4 & MAPK signaling & 0.65 & 0.11 & 0.57 & 0.05 & $F<M$ \\
\hline CRKL & & 0.66 & 0.13 & 0.59 & 0.16 & $F<M$ \\
\hline TRAF6 & & 0.30 & 0.18 & 0.73 & 0.11 & $F<M$ \\
\hline SLC6A1 & Transporter activity & 0.69 & 0.33 & 0.80 & 0.26 & $F<M$ \\
\hline$S L C 24 A 2$ & & 0.30 & 0.08 & 0.63 & 0.12 & $F<M$ \\
\hline SLC44A1 & & 0.71 & 0.30 & 0.30 & $<0.01$ & $F<M$ \\
\hline KCNIP1 & & 0.40 & 0.07 & 0.75 & 0.08 & $F<M$ \\
\hline TSC22D3 & & 0.56 & 0.15 & 0.58 & 0.07 & $\mathrm{~F}<\mathrm{M}$ \\
\hline HHIP & Brain development & 0.57 & 0.10 & 0.39 & 0.02 & $F<M$ \\
\hline SPRY4 & & 0.80 & 0.33 & 0.47 & 0.06 & $F<M$ \\
\hline MEISI & & 0.47 & $<0.01$ & 0.72 & 0.07 & $F<M$ \\
\hline ARCN1 & & 0.56 & 0.31 & 0.76 & 0.18 & $F<M$ \\
\hline DET1 & Protein assembly & 0.78 & 0.04 & 0.49 & 0.01 & $\mathrm{~F}<\mathrm{M}$ \\
\hline RBBP5 & & 0.38 & 0.02 & 0.70 & 0.03 & $F<M$ \\
\hline TRIM4 & & 0.71 & 0.14 & 0.46 & 0.32 & $F<M$ \\
\hline ZDHHC6 & Catalytic activity & 0.84 & 0.26 & 0.83 & 0.44 & $F<M$ \\
\hline$A B C F 2$ & & 0.51 & 0.05 & 0.52 & 0.10 & $F<M$ \\
\hline HELQ & & 0.60 & 0.18 & 0.69 & 0.35 & $F<M$ \\
\hline WDR33 & RNA processing & 0.69 & 0.16 & 0.66 & 0.21 & $F<M$ \\
\hline RBMS1 & & 0.59 & 0.34 & 0.78 & 0.05 & $F<M$ \\
\hline CLDND2 & Miscellaneous & 0.72 & 0.16 & 0.80 & 0.09 & $M>F$ \\
\hline$\angle R C H 1$ & & 0.84 & 0.16 & 0.85 & 0.31 & $F<M$ \\
\hline TM9SF1 & & 0.69 & 0.11 & 0.57 & 0.09 & $F<M$ \\
\hline ANKRD37 & & 0.69 & 0.05 & 0.62 & 0.23 & $F<M$ \\
\hline CAP1 & & 0.54 & 0.28 & 0.41 & 0.10 & $F<M$ \\
\hline
\end{tabular}

ventromedial gene expression profiles more closely resembled one another compared to ventrolateral prefrontal cortex. Dorsolateral prefrontal cortex is involved in working memory and cognitive control whereas ventral regions play a role in emotion regulation [50-52]. How prefrontal cortical gene expression profiles mediate behavioral functions remain to be explored but primate prefrontal profiles are spatially distinct.

Robust sex differences were identified across prefrontal cortical regions despite initial suggestions that sex differences in gene expression profiles are limited in human brain [17]. Recent results instead suggest that many genes are differentially expressed between the sexes for various brain regions in mice [18, 19], monkeys $[21]$, and humans $[9,22,23]$. Our results agree and confirm that 82 probes that targeted 80 unique genes are differentially expressed in male and female squirrel monkeys across prefrontal cortical regions.
Similar sex differences in different species raise the possibility of a conserved sexual signature in gene expression profiles of brain. Reinius et al. [21] found 85 genes differentially expressed between the sexes for occipital cortex in humans and cynomologus macaque monkeys. Only two of these genes were differentially expressed in occipital cortex of female and male marmoset monkeys [21]. These two genes were not examined in our study of squirrel monkeys but another gene in squirrel monkeys overlapped with the Reinius et al. [21] list for humans and macaques. In all three species, SLC6A1 is upregulated in cerebral cortex of females compared to males. Molecular genetic phylogenies suggest that macaques more closely resemble humans than do either squirrel monkeys or marmosets [53] but macaques and squirrel monkeys are polygamous and sexually dimorphic in size whereas marmoset males and females are monogamous and similar in size [54]. 
SLC6A1 encodes gamma-aminobutyric acid (GABA) transporter 1 (GAT-1) which terminates GABA neurotransmission via synaptic reuptake specifically in cerebral cortex [55]. Selective GAT-1 inhibitors such as tiagabine have anxiolytic and antidepressant effects in mice [56], and novel GAT-1 inhibitors are now being tested as potential treatments for anxiety and depression [57]. Studies of GAT-1 inhibitors have thus far focused primarily on males $[56,57]$, but our results suggest that sex differences warrant attention.

Another autosomal gene expressed at higher levels in squirrel monkey females compared to males across prefrontal cortical regions is HSD11B1. This gene encodes an enzyme that regulates glucocorticoid exposure by catalyzing conversion of inactive to active glucocorticoids [58]. A recent study of 134 healthy humans between the ages of 20 and 81 years reported increased skeletal muscle $H S D 11 B 1$ expression that correlated with age in women but not men [59]. We did not find significant correlations between age and HSD11B1 expression in any prefrontal cortical region for our smaller sample of female or male squirrel monkeys.

In humans with major depression, we recently identified FGF9 as a gene of interest [60]. Administration of $F G F 9$ to male rats increases anxiety and depression-like behavior [60], and FGF9 stimulates expression of SPRY4 in vitro [61]. In squirrel monkeys, FGF9 did not differ between the sexes but SPRY4 was increased across prefrontal cortical regions in females compared to males. SPRY4 encodes a negative regulator that suppresses neurotrophic functions [62, 63]. Inhibition of negative regulators that suppress neurotrophic functions may provide a novel approach for the development of new antidepressant medications [60].

Differential expression of single genes is noteworthy, but collective differences in multiple genes along functional pathways provide further insights [64]. Both MAPK and neurotrophin signaling pathways were significantly enriched with genes expressed at higher levels in squirrel monkey females compared to males across prefrontal cortical regions. More than half of the genes enriched in these pathways were associated with both MAPK and neurotrophin signaling. Neurotrophins activate MAPK signaling via extracellular signal-regulated kinases, and MAPK signaling is also activated by cJun $\mathrm{N}$-terminal kinases elicited by cellular stress and pro-inflammatory conditions [65]. These findings agree with evidence that women may be more vulnerable to the depressogenic effects of inflammation compared to men [66].

In human dorsal prefrontal cortex, the majority of genes differentially expressed between the sexes are autosomal and not sex chromosome genes [23]. Most sex differences in squirrel monkey prefrontal cortical regions are likewise autosomal, but two $\mathrm{X}$ chromosome genes were identified. Both SLC25A5 and TSC22D3 may have escaped X chromosome inactivation $[67,68]$ insofar as higher expression levels were discerned in squirrel monkey females compared to males across prefrontal cortical regions. TSC22D3 is induced by stress levels of glucocorticoids [69] and encodes a leucine zipper protein linked to MAPK signaling [70] and neuroplasticity in depression [71]. SLC25A5 encodes a mitochondrial protein [72] associated with anxiety in mice [73].

None of the stress main effects nor interactions in our study survived multiple testing correction possibly because of exposure to a mild intermittent social stressor. Nevertheless, effect sizes for sex differences were greater in stress compared to no-stress conditions for ventromedial and ventrolateral prefrontal cortical regions but not dorsolateral prefrontal cortex. Functional differences between regions suggest that stress modulates effect sizes for sex differences in prefrontal cortical regions involved in emotion regulation and not working memory nor cognitive control [50-52]. Two genes provide examples. Effect sizes for sex differences in SLC6A1 and SPRY4 expression were two to sevenfold greater in stress compared to no-stress conditions for ventromedial and ventrolateral prefrontal cortical regions. Increased SLC6A1 and SPRY4 expression in females compared to males during stressful conditions may respectively accelerate termination of GABA neurotransmission and inhibit neurotrophic functions. As discussed above, GABA neurotransmission and neurotrophic functions have been both implicated in mood and anxiety disorders $[56,60]$.

Greater effect sizes for sex differences in stress compared to no-stress conditions have additional implications for calls to include both sexes in preclinical animal research [74]. Studies not explicitly focused on stress often include manipulations or conditions that are inherently stressful. Our findings suggest that certain sex differences are amplified in stressful situations. If our findings from monkeys hold true for humans and other animals, then studies that include unintentional stressors with males alone may overlook significant sources of biological variability.

Our results should be interpreted in the context of potential limitations. Objectively similar stressors may be viewed differently by the sexes and small samples restricted our capacity to detect stress, sex, and region interactions. Although we analyzed 8853 probes that targeted 7299 unique genes, many probes designed for humans do not match the squirrel monkey genome and were not considered. Our array results require further validation, and bulk tissue samples do not necessarily reflect specific cell types or cellular stress states. Tissue samples were collected during non-breeding seasons when circulating sex steroid hormones remain stable at low levels, but organizational effects of sex steroids on brain development were not considered. 


\section{Conclusions}

Prefrontal cortical regions in squirrel monkey adults differed in the expression of numerous autosomal and sex chromosome genes. Robust sex differences were also identified across prefrontal cortical regions in the expression of mostly autosomal genes. Stress amplified sex difference effect sizes for gene expression profiles in prefrontal cortical regions involved in stress coping and emotion regulation. Results suggest new molecular targets for sex-specific treatments of stress disorders in human mental health.

\section{Additional files}

Additional file 1: Table S1. Human brain regions of interest. Specific regions of human frontal cortex and anterior cingulate cortex in Allen Brain Atlas array data (http://human.brain-map.org) that we compared with squirrel monkeys. (XLSX $11 \mathrm{~kb}$ )

Additional file 2: Table S2. Prefrontal cortical region effects. Genes targeted by probes with prefrontal cortical region main effects $(P<0.05)$ that survived multiple testing correction $(q<0.05)$. Data from 28 squirrel monkeys are presented for 5557 probes. Abbreviations: dIPFC dorosolateral prefrontal cortex, vIPFC ventrolateral prefrontal cortex, vmPFC ventromedial prefrontal cortex. (XLSX $852 \mathrm{~kb}$ )

Additional file 3: Table S3. Sex differences. Genes targeted by probes with sex difference main effects $(P<0.05)$ that survived multiple testing correction $(q<0.05)$ across squirrel monkey prefrontal cortical regions. Data from 10 males and 18 females are presented for 82 probes. (XLSX $22 \mathrm{~kb}$ )

Additional file 4: Table S4. Correlations between $\mathrm{QPCR}$ and array. Determinations by qPCR correlated with array results regardless of whether prefrontal cortical regions were analyzed separately (18 females +10 males $=28$ pairwise comparisons per region) or combined (3 regions $x$ [18 females +10 males $]=84$ pairwise comparisons). Abbreviations: dIPFC dorosolateral prefrontal cortex, vIPFC ventrolateral prefrontal cortex, vmPFC ventromedial prefrontal cortex. (XLSX $10 \mathrm{~kb}$ )

\section{Acknowledgements}

We are grateful to I. Birt, A. Pankonin, and D. Romero Vargas for help with array data processing.

\section{Funding}

This research was supported by National Institutes of Health MH47573 and the Pritzker Neuropsychiatric Disorders Research Consortium Fund LLC.

\section{Availability of data and materials}

Data are available for public use at https://pritzkerneuropsych.org/www/ data/.

\section{Authors' contributions}

DA collected the data. MH, KEM, and TLB analyzed the data. RMM, SJW, HA and AFS designed the study and interpreted data. AGL and DML worked on all aspects of this research. All authors read and approved the final manuscript.

\section{Ethics approval}

All procedures with animals were conducted in accordance with state and federal laws, standards of the Department of Health and Human Services, and were approved by the Institutional Animal Care and Use Committee at Stanford University.

\section{Competing interests}

Drs. Morrison and Bale report no biomedical financial interests or potential conflicts of interest related to this work. All other authors are members of the Pritzker Neuropsychiatric Disorders Research Consortium, which is supported by Pritzker Neuropsychiatric Disorders Research Fund, LLC. A shared intellectual property agreement exists between the academic and philanthropic entities of the Consortium. The Pritzker Neuropsychiatric Disorders Research Fund had no role in study design, data collection or analysis, decision to publish, or preparation of the manuscript.

\section{Publisher's Note}

Springer Nature remains neutral with regard to jurisdictional claims in published maps and institutional affiliations.

\section{Author details}

${ }^{1}$ Department of Psychiatry and Behavioral Sciences, Stanford University, 1201 Welch Rd MSLS Room P104, Stanford, CA 94305-5485, USA. ${ }^{2}$ Molecular and Behavioral Neuroscience Institute and Department of Psychiatry, University of Michigan, Ann Arbor, MI, USA. ${ }^{3}$ HudsonAlpha Institute for Biotechnology, Huntsville, AL, USA. ${ }^{4}$ Department of Animal Biology, University of Pennsylvania, Philadelphia, PA, USA.

Received: 25 July 2017 Accepted: 23 October 2017

Published online: 02 November 2017

\section{References}

1. Altemus M, Sarvaiya N, Neill EC. Sex differences in anxiety and depression clinical perspectives. Front Neuroendocrinol. 2014;35:320-30.

2. McHenry J, Carrier N, Hull E, Kabbaj M. Sex differences in anxiety and depression: role of testosterone. Front Neuroendocrinol. 2014;35:42-57.

3. Seedat S, Scott KM, Angermeyer MC, Berglund P, Bromet EJ, Brugha TS, Demyttenaere K, de Girolamo G, Haro JM, Jin R, et al. Cross-national associations between gender and mental disorders in the World Health Organization World Mental Health Surveys. Arch Gen Psychiatry. 2009;66:785-95.

4. Bale TL, Epperson CN. Sex differences and stress across the lifespan. Nat Neurosci. 2015:18:1413-20.

5. Oikonomidis L, Santangelo AM, Shiba Y, Clarke FH, Robbins TW, Roberts AC. A dimensional approach to modeling symptoms of neuropsychiatric disorders in the marmoset monkey. Dev Neurobiol. 2017;77:328-53.

6. Lueken U, Hahn T. Functional neuroimaging of psychotherapeutic processes in anxiety and depression: from mechanisms to predictions. Curr Opin Psychiatry. 2016:29:25-31.

7. McEwen BS, Morrison JH. The brain on stress: vulnerability and plasticity of the prefrontal cortex over the life course. Neuron. 2013;79:16-29.

8. Beery AK, Zucker I. Sex bias in neuroscience and biomedical research. Neurosci Biobehav Rev. 2011;35:565-72

9. Trabzuni D, Ramasamy A, Imran S, Walker R, Smith C, Weale ME, Hardy J, Ryten M. North American brain expression C. Widespread sex differences in gene expression and splicing in the adult human brain. Nat Commun. 2013:4:2771

10. Hawrylycz M, Miller JA, Menon V, Feng D, Dolbeare T, Guillozet-Bongaarts $A L$, Jegga $A G$, Aronow BJ, Lee CK, Bernard $A$, et al. Canonical genetic signatures of the adult human brain. Nat Neurosci. 2015:18:1832-44.

11. Bernard A, Lubbers LS, Tanis KQ, Luo R, Podtelezhnikov AA, Finney EM, McWhorter MM, Serikawa K, Lemon T, Morgan R, et al. Transcriptional architecture of the primate neocortex. Neuron. 2012;73:1083-99.

12. Fukuoka T, Sumida K, Yamada T, Higuchi C, Nakagaki K, Nakamura K, Kohsaka S, Saito K, Oeda K. Gene expression profiles in the common marmoset brain determined using a newly developed common marmoset-specific DNA microarray. Neurosci Res. 2010;66:62-85.

13. Ochsner KN, Silvers JA, Buhle JT. Functional imaging studies of emotion regulation: a synthetic review and evolving model of the cognitive control of emotion. Ann N Y Acad Sci. 2012;1251:E1-24.

14. Ray $\mathrm{RD}$, Zald $\mathrm{DH}$. Anatomical insights into the interaction of emotion and cognition in the prefrontal cortex. Neurosci Biobehav Rev. 2012;36:479-501.

15. Collins KA, Mendelsohn A, Cain CK, Schiller D. Taking action in the face of threat: neural synchronization predicts adaptive coping. J Neurosci. 2014;34:14733-8.

16. McKlveen JM, Myers B, Herman JP. The medial prefrontal cortex: coordinator of autonomic, neuroendocrine and behavioural responses to stress. Jeuroendocrinol. 2015;27:446-56

17. Rinn $J$, Snyder M. Sexual dimorphism in mammalian gene expression. Trends Genet. 2005:21:298-305.

18. Yang X, Schadt EE, Wang S, Wang H, Arnold AP, Ingram-Drake L, Drake TA, Lusis AJ. Tissue-specific expression and regulation of sexually dimorphic genes in mice. Genome Res. 2006;16:995-1004. 
19. Vied C, Ray S, Badger CD, Bundy JL, Arbeitman MN, Nowakowski RS. Transcriptomic analysis of the hippocampus from six inbred strains of mice suggests basis for sex-specific susceptibility and severity of neurological disorders. J Comp Neurol. 2016;524:2696-710.

20. Morgan CP, Bale TL. Sex differences in microRNA regulation of gene expression: no smoke, just miRs. Biol Sex Differ. 2012;3:22.

21. Reinius B, Saetre P, Leonard JA, Blekhman R, Merino-Martinez R, Gilad Y, Jazin $\mathrm{E}$. An evolutionarily conserved sexual signature in the primate brain. PLoS Genet. 2008;4:e1000100.

22. Xu H, Wang F, Liu Y, Yu Y, Gelernter J, Zhang H. Sex-biased methylome and transcriptome in human prefrontal cortex. Hum Mol Genet. 2014;23:1260-70.

23. Mayne BT, Bianco-Miotto T, Buckberry S, Breen J, Clifton V, Shoubridge C, Roberts $C T$. Large scale gene expression meta-analysis reveals tissue-specific, sex-biased gene expression in humans. Front Genet. 2016;7:183.

24. Crews D. Diversity of hormone-behavior relations in reproductive behavior. In: Becker JB, Breedlove SM, Crews D, McCarthy MM, editors. Behavioral endocrinology. 2nd ed. Cambridge: MIT Press; 2002. p. 223-87.

25. Palanza P, Parmigiani S. How does sex matter? Behavior, stress and animal models of neurobehavioral disorders. Neurosci Biobehav Rev. 2017;76(Pt A):134-43.

26. Mendoza SP, Lyons DM, Saltzman W. Sociophysiology of squirrel monkeys. Am J Primatol. 1991;23:37-54.

27. Mendoza SP, Hennessy MB, Lyons DM. Distinct immediate and prolonged effects of separation on plasma cortisol in female squirrel monkeys. Psychobiology. 1992;20:300-6.

28. Karssen AM, Her S, Li JZ, Patel PD, Meng F, Bunney WE Jr, Jones EG, Watson SJ, Akil H, Myers RM, et al. Stress-induced changes in primate prefrontal profiles of gene expression. Mol Psychiatry. 2007;12:1089-102.

29. Lee AG, Buckmaster CL, Yi E, Schatzberg AF, Lyons DM. Coping and glucocorticoid receptor regulation by stress inoculation. Psychoneuroendocrinology. 2014;49:272-9.

30. Lyons DM, Buckmaster PS, Lee AG, Wu C, Mitra R, Duffey LM, Buckmaster $\mathrm{CL}$, Her S, Patel PD, Schatzberg AF. Stress coping stimulates hippocampal neurogenesis in adult monkeys. Proc Natl Acad Sci U S A. 2010;107: 14823-7.

31. Akil H. Scientific strategy in neuroscience: discovery science versus hypothesis-driven research. Neurosci Quarterly. 2003; Spring:4-5, https:// www.sfn.org/news-and-calendar/neuroscience-quarterly/past-issues. Accessed 1 Oct 2017.

32. Quackenbush J. Computational approaches to analysis of DNA microarray data. Yearb Med Inform. 2006;45:91-103.

33. Lyons DM, Mendoza SP, Mason WA. Sexual segregation in squirrel monkeys (Saimiri sciureus): a transactional analysis of adult social dynamics. J Comp Psychol. 1992;106:323-30.

34. Patel PD, Katz M, Karssen AM, Lyons DM. Stress-induced changes in corticosteroid receptor expression in primate hippocampus and prefrontal cortex. Psychoneuroendocrinology. 2008;33:360-7.

35. Schiml PA, Mendoza SP, Saltzman W, Lyons DM, Mason WA. Annual physiological changes in individually housed squirrel monkeys (Saimiri sciureus). Am J Primatol. 1999;47:93-103.

36. Gergen JA, MacLean PD. A stereotaxic atlas of the squirrel monkey's brain (Saimiri sciureus) vol. 933. Bethesda: Public Health Service; 1962.

37. Thornton B, Basu C. Real-time PCR ( $(\mathrm{PPCR})$ primer design using free online software. Biochem Mol Biol Educ. 2011;39:145-54.

38. Livak KJ, Schmittgen TD. Analysis of relative gene expression data using real-time quantitative PCR and the 2(-Delta Delta C(T)) method. Methods. $2001 ; 25: 402-8$

39. Kent WJ. BLAT—-the BLAST-like alignment tool. Genome Res. 2002;12:656-64.

40. Core Team R. R: a language and environment for statistical computing. Vienna. Austria: R Foundation for Statistical Computing; 2013.

41. Xie $Y$, Wang $X$, Story M. Statistical methods of background correction for Illumina BeadArray data. Bioinformatics. 2009;25:751-7.

42. Maxwell SE, Delaney HD. Designing experiments and analyzing data: a model comparison perspective. 2nd ed. Mahwah: Lawerence Erlbaum Associates; 2004. p. 763-820

43. Lu T, Pan Y, Kao SY, Li C, Kohane I, Chan J, Yankner BA. Gene regulation and DNA damage in the ageing human brain. Nature. 2004;429:883-91.

44. Storey JD, Tibshirani R. Statistical significance for genomewide studies. Proc Natl Acad Sci U S A. 2003;100:9440-5.

45. Bates D, Machler M, Bolker B, Walker S. Fitting linear mixed-effects models using Ime4. J Stat Softw. 2015;67:1-48.
46. Hawrylycz MJ, Lein ES, Guillozet-Bongaarts AL, Shen EH, Ng L, Miller JA, van de Lagemaat LN, Smith KA, Ebbert A, Riley ZL, et al. An anatomically comprehensive atlas of the adult human brain transcriptome. Nature. 2012:489:391-9.

47. Huang da W, Sherman BT, Lempicki RA. Systematic and integrative analysis of large gene lists using DAVID bioinformatics resources. Nat Protoc. 2009;4:44-57.

48. Lakens D. Calculating and reporting effect sizes to facilitate cumulative science: a practical primer for t-tests and ANOVAs. Front Psychol. 2013;4:863.

49. Becker JB, Prendergast BJ, Liang JW. Female rats are not more variable than male rats: a meta-analysis of neuroscience studies. Biol Sex Differ. 2016;7:34.

50. Mitchell DG. The nexus between decision making and emotion regulation: a review of convergent neurocognitive substrates. Behav Brain Res. 2011;217:215-31.

51. Smolker HR, Depue BE, Reineberg AE, Orr JM, Banich MT. Individual differences in regional prefrontal gray matter morphometry and fractional anisotropy are associated with different constructs of executive function. Brain Struct Funct. 2015;220:1291-306.

52. Mueller SC. The influence of emotion on cognitive control: relevance for development and adolescent psychopathology. Front Psychol. 2011;2:327.

53. Shoshani J, Groves CP, Simons EL, Gunnell GF. Primate phylogeny: morphological vs. molecular results. Mol Phylogenet Evol. 1996;5:102-54.

54. Smuts BR, Cheney DL, Seyfarth RM, Wrangham RW, Struhsaker TT. Primate societies. Chicago: University of Chicago Press; 1986.

55. Conti F, Minelli A, Melone M. GABA transporters in the mammalian cerebral cortex: localization, development and pathological implications. Brain Res Brain Res Rev. 2004;45:196-212.

56. Thoeringer CK, Erhardt A, Sillaber I, Mueller MB, Ohl F, Holsboer F, Keck ME. Long-term anxiolytic and antidepressant-like behavioural effects of tiagabine, a selective GABA transporter-1 (GAT-1) inhibitor, coincide with a decrease in HPA system activity in C57BL/6 mice. J Psychopharmacol. 2010;24:733-43.

57. Salat K, Podkowa A, Malikowska N, Kern F, Pabel J, Wojcieszak E, Kulig K, Wanner KT, Strach B, Wyska E. Novel, highly potent and in vivo active inhibitor of GABA transporter subtype 1 with anticonvulsant, anxiolytic, antidepressant and antinociceptive properties. Neuropharmacology. 2017;113(Pt A):331-42.

58. Wyrwoll CS, Holmes MC, Seckl JR. 11 beta-hydroxysteroid dehydrogenases and the brain: from zero to hero, a decade of progress. Front Neuroendocrinol 2011;32:265-86.

59. Hassan-Smith ZK, Morgan SA, Sherlock M, Hughes B, Taylor AE, Lavery GG, Tomlinson JW, Stewart PM. Gender-specific differences in skeletal muscle 11 beta-HSD1 expression across healthy aging. J Clin Endocrinol Metab. 2015;100:2673-81.

60. Aurbach EL, Inui EG, Turner CA, Hagenauer MH, Prater KE, Li JZ, Absher D, Shah N, Blandino P Jr, Bunney WE, et al. Fibroblast growth factor 9 is a novel modulator of negative affect. Proc Natl Acad Sci U S A. 2015;112:11953-8.

61. Sylvestersen KB, Herrera PL, Serup P, Rescan C. FGF9 signalling stimulates Spred and Sprouty expression in embryonic mouse pancreas mesenchyme. Gene Expr Patterns. 2011;11:105-11.

62. Katoh Y, Katoh M. FGF signaling inhibitor, SPRY4, is evolutionarily conserved target of WNT signaling pathway in progenitor cells. Int J Mol Med. 2006;17:529-32.

63. Kim HJ, Bar-Sagi D. Modulation of signalling by Sprouty: a developing story. Nat Rev Mol Cell Biol. 2004;5:441-50.

64. Subramanian A, Tamayo P, Mootha VK, Mukherjee S, Ebert BL, Gillette MA, Paulovich A, Pomeroy SL, Golub TR, Lander ES, et al. Gene set enrichment analysis: a knowledge-based approach for interpreting genome-wide expression profiles. Proc Natl Acad Sci U S A. 2005;102:15545-50.

65. Cargnello M, Roux PP. Activation and function of the MAPKs and their substrates, the MAPK-activated protein kinases. Microbiol Mol Biol Rev. 2011;75:50-83.

66. Bekhbat M, Neigh GN. Sex differences in the neuro-immune consequences of stress: focus on depression and anxiety. Brain Behav Immun. 2017; doi. org/10.1016/j.bbi.2017.02.006

67. Carrel L, Willard HF. X-inactivation profile reveals extensive variability in Xlinked gene expression in females. Nature. 2005;434:400-4.

68. Peeters SB, Cotton AM, Brown CJ. Variable escape from X-chromosome inactivation: identifying factors that tip the scales towards expression. BioEssays. 2014;36:746-56.

69. Ayroldi E, Riccardi C. Glucocorticoid-induced leucine zipper (GILZ): a new important mediator of glucocorticoid action. FASEB J. 2009;23:3649-58.

70. Clark AR, Lasa M. Crosstalk between glucocorticoids and mitogenactivated protein kinase signalling pathways. Curr Opin Pharmacol. 2003;3:404-11. 
71. Frodl T, Carballedo A, Hughes MM, Saleh K, Fagan A, Skokauskas N, McLoughlin DM, Meaney J, O'Keane V, Connor TJ. Reduced expression of glucocorticoidinducible genes GILZ and SGK-1: high IL-6 levels are associated with reduced hippocampal volumes in major depressive disorder. Transl Psychiatry. 2012;2:e88.

72. Clemencon B, Babot M, Trezeguet V. The mitochondrial ADP/ATP carrier (SLC25 family): pathological implications of its dysfunction. Mol Asp Med. 2013;34:485-93.

73. Filiou MD, Zhang Y, Teplytska L, Reckow S, Gormanns P, Maccarrone G, Frank E, Kessler MS, Hambsch B, Nussbaumer M, et al. Proteomics and metabolomics analysis of a trait anxiety mouse model reveals divergent mitochondrial pathways. Biol Psychiatry. 2011;70:1074-82.

74. McCullough LD, de Vries GJ, Miller VM, Becker JB, Sandberg K, McCarthy MM. NIH initiative to balance sex of animals in preclinical studies: generative questions to guide policy, implementation, and metrics. Biol Sex Differ. 2014;5:15.

Submit your next manuscript to BioMed Central and we will help you at every step:

- We accept pre-submission inquiries

- Our selector tool helps you to find the most relevant journal

- We provide round the clock customer support

- Convenient online submission

- Thorough peer review

- Inclusion in PubMed and all major indexing services

- Maximum visibility for your research

Submit your manuscript at www.biomedcentral.com/submit
Biomed Central 